\title{
Dimensionen der Führung: Eine begriffsdefinitorische Grundlegung (ethikorientierter) guter Führung
}

\author{
Lisa Schmalzried ${ }^{1,2}\left(\right.$ D $\cdot$ Friederike Fröhlich ${ }^{2} \cdot$ Marcel Vondermaßen $^{3}$
}

Received: 21 July 2021 / Accepted: 30 November 2021 / Published online: 3 January 2022

(c) The Author(s) 2022, korrigierte Publikation 2022

\begin{abstract}
Angesichts der Omnipräsenz von Führung, ihres Potentials und leider auch des häufigen Missbrauches von Führung stellt sich die Frage, was gute Führung auszeichnet. Als Antwort hierauf werden seit den 1980er Jahren vermehrt führungsethische Theorien formuliert. Diese Ansätze zeichnen sich allerdings durch eine große Heterogenität aus. Das ist sowohl eine Herausforderung für die wissenschaftlich-theoretische Debatte als auch für ihren praxisbezogenen Orientierungsanspruch. Dieser Artikel setzt hier an und formuliert eine Minimaldefinition, die alle zentralen Dimensionen von Führung erfasst, sich trotzdem auf das Wesentliche konzentriert, zugleich normativ neutral ist als auch moralische Fragen mitdenkt: Führung ist ein interpersoneller, asymmetrischer, kontextsensibler und zielorientierter Prozess der Einflussnahme. Diese Definition zeigt, dass Führung fünf miteinander verflochtene aber analytisch trennbare Dimensionen aufweist: (1) Führende, (2) Geführte (3) Interaktionsprozess (4) Ziele und (5) Kontext. Damit ist ein gemeinsamer Bezugsrahmen der Debatte vorhanden, der die wesentlichen Dimensionen von Führung und ihre moralischen Orte theorieübergreifend identifiziert. So können unterschiedliche (ethische) Führungstheorien miteinander ins Gespräch kommen, miteinander verglichen und bewertet werden. Zugleich ist damit ein Fundament für die potentielle Weiterentwicklung und moraltheoretische Fundierung führungsethischer Theorien geschaffen.
\end{abstract}

Schlagwörter Minimaldefinition der Führung · (ethische) Dimensionen der Führung · Postindustrielle Führung · Transformationale Führung · Ethische Führung · Verantwortungsvolle Führung

\section{Hinführung}

Führung ist allgegenwärtig-in der Politik, in Bildungsinstitutionen, in Wirtschaftsunternehmen, in gemeinnützigen Organisationen oder in Vereinen, um nur einige Beispiele zu nennen. Sie ist teilweise innerhalb von formellen Kontexten mit klar definierten Rollenverständnissen zu beobachten, teilweise auch in informellen Settings, wie

Lisa Schmalzried

lisa.schmalzried@wcge.org

Friederike Fröhlich

friederike.froehlich@wcge.org

Marcel Vondermaßen

marcel.vondermassen@izew.uni-tuebingen.de

1 Martin-Luther-Universität Halle-Wittenberg, Halle, Germany

2 Wittenberg-Zentrum für Globale Ethik, Lutherstadt Wittenberg, Germany

3 Internationales Zentrum für Ethik in den Wissenschaften, Tübingen, Germany zwischen Freund*innen, in Familien oder Beziehungen. Häufig, wenn Menschen zusammenkommen, kristallisiert sich ein Führungsprozess heraus. Führung erscheint als zwischenmenschliche Konstante. Dass dem so ist, kann man dadurch erklären, dass man durch gute, gelungene Führung gemeinsam mehr erreichen kann. Führung gibt Gruppen Orientierung, kann ein Wir-Gefühl fördern und koordiniert Ziele, Aufgaben und Verantwortlichkeiten.

Angesichts der Omnipräsenz von Führung, ihres Potentials und leider auch des häufigen Missbrauches oder Scheiterns von Führung ${ }^{1}$ stellt sich die Frage, was gute Führung auszeichnet. Als Antwort hierauf werden seit den 1980er Jahren vermehrt führungsethische Theorien formuliert. Man denke bspw. an die Theorien der ethischen Führung (Brown et al. 2005; Brown und Treviño 2006), verantwortlichen Führung (Maak und Pless 2006), werteorientierten Führung (Frey und Schmalzried 2013), transformationellen Führung (Bass 1985; Burns 1978), spirituellen Führung (Fry 2003), dienenden Führung (Greenleaf 1977; Van Dierendonck 2011) oder authentischen Führung (Avolio 1 Vgl. bspw. den wachsenden Diskurs zur destruktiven Führung Mackey et al. (2020) sowie Schyns und Schilling (2013). 
und Gardner 2005). Der gemeinsame Grundgedanke dieser sonst durchaus unterschiedlichen Ansätze ist, dass der Erfolg von Führung (auch) anhand eines moralischen Maßstabes beurteilt werden sollte. Trotz dieser gemeinsamen Grundannahme lässt sich eine große Heterogenität bei den bestehenden führungsethischen Theorien beobachten. Zum einen gibt es explizit führungsethische Theorien, die es sich bewusst zum Ziel setzen, gute Führung als moralisch gute Führung zu beschreiben (vgl. z. B. Brown et al. 2005; Maak und Pless 2006; Pless und Maak 2011; Burns 1978; Frey und Schmalzried 2013) und implizite Theorien, die in ihrer Definition von guter Führung auch Kriterien aufnehmen, die man als moralische ansehen kann (vgl. z. B. Avolio und Gardner 2005; Bass 1985; Greenleaf 1977). Auch rechtfertigen die Theorien die Relevanz von Moral unterschiedlich, teils instrumentell (vgl. z. B. Brown und Treviño 2006), teils intrinsisch (vgl. Pless und Maak 2011). Oder sie sind sich uneins darüber, welche Aspekte von Führung moralisch relevant sind und konzentrieren sich somit auf unterschiedliche Führungsdimensionen und deren moralische Fragen, wie die Ausführungen im Abschnitt 3 zeigen werden. ${ }^{2}$

Ein Problem der führungsethischen Debatte und sicherlich auch eine Erklärung ihrer Heterogenität besteht in dem Umstand, dass deren Protagonist*innen nicht genau bzw. nicht einheitlich definieren, worüber sie sprechen. Schon Anfang der 1990er Jahre beklagte Rost (1993), dass viele Führungstheorien keine grundlegende, theorieübergreifende Definition von Führung angeben. Diese Kritik wurde von Winston und Patterson (2006) erneut aufgegriffen. Diese Lücke will Rost mit seiner postindustriellen Führungsdefinition und Winston und Patterson mit ihrer integrativen Führungsdefinition füllen. Auch wenn diese Autoren eine gute Übersicht über unterschiedliche Führungsdefinitionen liefern und auf verschiedene Aspekte von Führung verweisen, so ist eines der Hauptprobleme ihrer Definitionen, dass sie nicht normativ neutral sind. Diese Kritik trifft auch auf viele Definitionen innerhalb der führungsethischen Debatte zu (vgl. bspw. für weitere bereits normativ aufgeladene Führungsdefinitionen Burns 1978; Faix et al 2020; Fairhurst und Uhl-Bien 2012; Greenleaf 2013; Kort 2008; Luthans und Avolio 2003). Will man aber verstehen, was

\footnotetext{
${ }^{2}$ Vgl. für eine ähnliche Diagnose der theoretischen Konfusion und definitorischen Ambiguität der führungsethischen Debatte auch Lemoine et al. (2019) und ihren Vergleich der ethischen, authentischen und dienenden Führung. Sie wollen diese Theorien konzeptionell schärfen, indem sie deren moraltheoretischen Hintergründe herausarbeiten. Damit gehen sie unserer Ansicht den zweiten Schritt vor dem ersten Schritt: Auch wenn wir die moraltheoretische Fundierung von Führungstheorien für wichtig erachten, braucht es erst ein grundsätzliches Verständnis von Führung, um Theorien vergleichen und bewerten, sowie die Auswahl bestimmter Führungsaspekte motivieren und dabei gleichzeitig in ein größeres Bild einordnen zu können.
}

gute Führung ist, so erscheint es naheliegend, sich zunächst darüber zu verständigen, was rein deskriptiv überhaupt Führung ist, um sich erst in einem zweiten Schritt darauf zu konzentrieren, wie sich diese bewerten lässt. ${ }^{3}$ Mit diesem Vorgehen kann man ein besseres Verständnis von Führung entwickeln und zwar von guter, sowie von schlechter Führung. Ohne solch ein normativ neutrales Verständnis von Führung können unseres Erachtens unterschiedliche Führungstheorien nicht bzw. nur schwer miteinander ins Gespräch kommen, miteinander verglichen und bewertet werden. Dies gilt sowohl für den Vergleich von unterschiedlichen führungsethischen Theorien untereinander als auch den Vergleich von führungsethischen mit anderen normativen Führungstheorien. Außerdem kann man ausgehend von einer grundlegenden Führungsdefinition auch leichter erkennen, warum Moral für Führung eine Rolle spielt und wo genau „Orte der Moral“4 im Bereich der Führung zu finden sind. Hiermit ist auch ein Fundament für die potenzielle Weiterentwicklung und moraltheoretische Fundierung führungsethischer Theorien geschaffen.

Ziel dieses Artikels ist es deshalb eine grundlegende Definition von Führung zu erarbeiten als möglichst umfassenden und zugleich debattenstrukturierenden, theorieunabhängigen und normativ neutralen Bezugsrahmen. Wir haben das Ziel, eine Minimaldefinition der Führung zu formulieren, die alle wesentlichen Dimensionen erfasst, sich trotzdem auf das Wesentliche konzentriert, zugleich normativ neutral ist als auch moralische Fragen mitdenkt. Dafür wird im Abschnitt 1 eine möglichst wertneutrale Minimaldefinition von Führung eingeführt, die Führung als interpersonellen asymmetrischen, zielorientierten und kontextsensiblen Prozess der Einflussnahme versteht. Im Abschnitt 2 wird gezeigt, dass die Führungsdefinition alle vier

\footnotetext{
${ }^{3}$ Für Ciulla stellt dies kein Problem dar. Was Führung ist, ist für sie nicht die eigentlich interessante Frage, sondern die, was gute Führung ist. Uneinigkeit entstünde auch erst bei der zweiten Frage, so Ciulla, nicht bei der ersten: „Leadership does not denote radically different things for different scholars. One can detect a family resemblance between the different definitions. All of them talk about leadership as some kind of process, act, or influence that in some way gets people to do something." (Ciulla 1995). Man kann Ciulla recht geben, dass die Frage, was Führung ist, letztendlich von Interesse ist, da man an der Frage interessiert ist, was gute Führung auszeichnet. Auch sei unbestritten, dass ein Großteil der Diskussion und Uneinigkeit im Hinblick auf die zweite Frage aufkommt. Dennoch halten wir es für hilfreich, mit einer grundlegenden Definition von Führung zu beginnen, um ausgehend von einem gemeinsamen Verständnis die Frage nach guter Führung zu stellen. Nur so können wir sicher gehen, alle relevanten Aspekte von Führung zu erfassen.

4 ,Orte der Moral ' meint, dass sich in den einzelnen Dimensionen von Führung, aber auch in Spannungsfeldern zwischen Ihnen, moralische Fragestellungen ergeben. Diese können sowohl für Reflexionen über gute Führung von Bedeutung sein als auch in der täglichen Praxis.
} 
Adäquatheitsbedingungen erfüllt, die an eine solche Definition gestellt werden können. Abschnitt 3 vergleicht diese Definition mit drei prominenten Führungsverständnissen der (führungsethischen) Debatte und zeigt auf, dass und wie diese Definition dabei hilft, bestehende (ethikorientierte) Theorien der Führung differenziert zu analysieren und zu bewerten.

\section{Minimaldefinition der Führung}

Dieser Artikel setzt an folgender Annahme an: Eine grundlegende Definition von Führung, also eine Minimaldefinition, ist notwendig, um ein besseres Verständnis von Führung zu entwickeln und unterschiedliche Theorien und Verständnisse von Führung beurteilen, miteinander vergleichen und gegebenenfalls selbst eine solche Theorie erarbeiten zu können. Zudem ist ein solches Verständnis die Basis für alle führungsethischen Theorien, um sicherzustellen, dass man alle relevanten Dimensionen von Führung erfasst, und um zu erkennen, wo sich normative Fragen im Bereich der Führung stellen.

Eine solche grundlegende Definition könnte man versuchen durch eine Begriffsanalyse von ,Führung ‘ zugewinnen. Hier stellt sich jedoch das Problem, dass sich unsere alltäglichen Sprachintuitionen und die theoretischen Ansätze zum Begriff der Führung als äußerst heterogen erweisen. ,Führung ' ist ein mehrdeutiger und vager Begriff. Daher bietet sich unseres Erachtens zur Erarbeitung einer Minimaldefinition der Führung eher die Methode einer Begriffsexplikation denn die der Begriffsanalyse an. Eine Begriffsexplikation verfolgt gerade das Ziel, einen vagen Begriff durch einen exakteren zu ersetzen (vgl. Carnap 1958).

Die sich aus einer Begriffsexplikation ergebende Führungsdefinition sollte dabei folgende Adäquatheitsbedingungen erfüllen: (1) Sie sollte weit genug gefasst sein, um unterschiedlichen alltäglichen und theoretischen Führungsverständnissen bestmöglich gerecht zu werden. Diese Bedingung ist wichtig, damit die Minimaldefinition ihre diskussionsstrukturierende Aufgabe erfüllt und man auf ihrer Basis unterschiedliche Führungstheorien miteinander vergleichen kann. (2) Verbunden hiermit sollte die Minimaldefinition insoweit normativ neutral sein, als dass sie gelungene, ebenso wie schlechte Führung erfassen kann. In Bezug auf führungsethische Theorien sollte eine Minimaldefinition somit auch moralisch schlechte Führung als Führung anerkennen. Dies ist insofern von Bedeutung, da viele Theorien unterschiedlich normativ aufgeladene Führungsbegriffe verwenden. (3) Im Hinblick auf führungsethische Theorien ist es außerdem wichtig, dass die Minimaldefinition aufzeigt, wo sich im Bereich der Führung moralische Fragen stellen, anders formuliert sollte sie auf ,Orte der Moral' innerhalb von Führung verweisen. Dies ist entscheidend, da hierüber geprüft werden kann, ob eine führungsethische Theorie alle relevanten moralischen Dimensionen von Führung adressiert. Dies ist insbesondere auch wichtig, da die moralischen Fragestellungen, die sich im Bereich der Führung stellen, teilweise auch dimensionsübergreifende Fragen sind. (4) Zugleich sollte sie sich noch auf keine Moraltheorie festlegt haben, um mit unterschiedlichen führungsethischen Theorien kompatibel zu sein.

Unseres Erachtens erfüllt die folgende Definition diese Adäquatheitsbedingungen: ${ }^{5}$

$\mathrm{DF}_{\text {minimal }}$ : Führung ist ein asymmetrischer Prozess, der in einem Kontext $\mathrm{K}$ zwischen mindestens zwei Personen X (Führende) und Y (Geführte) stattfindet, so dass $\mathrm{X}$ durch Mittel $\mathrm{M}$ eine von $\mathrm{X}$ intendierte ausschlagebene Rolle dabei spielt, dass Y Ziele Z akzeptiert, oder dass X durch Mittel $\mathrm{M}$ eine ausschlaggebende Rolle dabei spielt, das Y bei der Umsetzung von $\mathrm{Z}$ mitwirkt.

$\mathrm{DF}_{\text {minimal }}$ versteht Führung als interpersonellen, asymmetrischen und kontextsensiblen Prozess der Einflussnahme im Hinblick auf Ziele und Handlungen. Hierbei lässt $\mathrm{DF}_{\text {minimal }}$ fünf analytisch voneinander trennbare, aber dennoch miteinander verbundene Dimensionen von Führung erkennen:

(1) Führende $X^{6}$

(2) Geführte Y

(3) Zwischenmenschlicher Interaktionsprozess, insbesondere der Einsatz der Mittel M

(4) Ziele Z (umfasst, sowohl Zielauswahl als auch die Zielumsetzung)

(5) Kontext K

Ob Führung als interpersoneller Prozess zustande kommt, hängt von allen Beziehungspartner*innen ab, also von Führenden als auch Geführten, ebenso wie dem jeweiligen Handlungskontext, also beispielsweise von strukturellen,

\footnotetext{
${ }^{5}$ Für die Erarbeitung dieser Definition haben wir uns von unseren mehrjährigen Auseinandersetzungen mit alltäglichen Führungsprozessen auf der einen Seite und mit philosophischen, psychologischen und ökonomischen Führungsdefinitionen leiten lassen. Die dabei von uns identifizierten Kerndimensionen von Führung haben wir mit zentralen Arbeiten zu Führungsdefinitionen (vgl. insbesondere Rost (1991), Ciulla (1995), Winston und Patterson (2006)) sowie den oben aufgeführten führungsethischen Theorien abgeglichen und mit den hier dargestellten Adäquatheitsbedingungen an Definitionen evaluiert, siehe hierzu auch Abschnitt 3.

${ }^{6}$ Wir verwenden im Text bewusst die Bezeichnung „Führende“. Im Englischen würden wir von „Leaders“ sprechen. Die Bezeichnung „Führer*in“ kann man durch die deutsche Geschichte des Nationalsozialismus nicht mehr verwenden. Führungskraft" bezieht sich auf eine Positionsbezeichnung innerhalb einer hierarchischen Organisation, „Führungspersönlichkeit“" stellt mehr die Eignung, Führungsaufgaben zu übernehmen in den Vordergrund.
} 
institutionellen oder gesellschaftlichen Bedingungen. Sich einseitig auf die Führenden, deren Charaktereigenschaften und Handlungen zu konzentrieren, wie es so viele traditionelle Führungstheorien, allen voran die, great man' Theorien, tun, ist somit nicht empfehlenswert.

Nichtsdestotrotz ist Führung ein asymmetrischer Prozess: Im Hinblick auf die Ziele $\mathrm{Z}$ beeinflussen die Führenden $\mathrm{X}$ die Geführten Y durch die Mittel M. Ob und wie fluide diese Asymmetrie ist, hängt vom jeweiligen Organisationstyp ab. In allen Führungsprozessen gilt aber: Durch den Einsatz der Mittel M gibt X Y Gründe an die Hand, die Ziele Z oder deren Umsetzung zu akzeptieren. Wie nachfolgend noch genauer ausgeführt wird, haben wir ein weites Verständnis davon, was ein Mittel M sein kann. Dies umfasst eher instrumentelle Mittel, wie geldwerte Vorteile, aber auch Eigenschaften wie Charisma und Überzeugungskraft. Diese Richtung der Einflussnahme lässt sich nicht umdrehen, ohne den Führungsprozess umzudrehen. Damit ist nicht gesagt, dass Geführte Führende überhaupt nicht beeinflussen können. Geführte können auf vielerlei Weise Einfluss auf Führende nehmen, beispielsweise durch Zuarbeit, Unterstützung, Interesse an Projekten oder auch Kritik bis hin zu aktivem oder passivem Widerstand oder den Versuch selbst zu führen. Letztlich führt aber die Person, der es gelingt, andere zielorientiert zu beeinflussen. Die Rolle von $X$ als Führenden und $\mathrm{Y}$ als Geführten sind aber nicht zwingend dauerhaft festgelegt. Die Einflussnahme kann sich unter Umständen umdrehen oder auflösen (vgl. z. B. Rost 1991).

\section{Erfüllung der Adäquatheitsbedingungen durch die Minimaldefinition der Führung}

Im Folgenden wollen wir zeigen, dass $\mathrm{DF}_{\text {minimal }}$ den vier Adäquatheitsbedingungen gerecht wird. Dass sie Adäquatheitsbedingung 1 erfüllt und weit genug gefasst ist, um unterschiedliche Führungsverständnisse und Schwerpunkte abzudecken und so auch hilft, diese zu vergleichen, soll die nachfolgende Auflistung veranschaulichen. Zur besseren Übersichtlichkeit orientiert sich die Auflistung dabei thematisch an den Führungsdimensionen:

\section{Führende X/Geführte Y}

a) Laut $\mathrm{DF}_{\text {minimal }}$ kann Führung ein Prozess zwischen Individuen, wie beispielsweise Führungskräften und Mitarbeiter*innen, zwischen Individuen und Gruppen, wie beispielsweise Führungskräften und Teams, oder zwischen Gruppen, also beispielsweise Vorständen und Teams, sein.

b) Die Führenden müssen nicht direkt beabsichtigen, eine Führungsrolle zu übernehmen. Das kann beispielsweise passieren, wenn ein*e Mitarbeiter*in durch Initiative und Engagement ein Projekt voranbringt und Kolleg*innen dafür begeistert. Sein*ihr Fokus liegt dann primär auf der Umsetzung des Projekts und nicht auf der Übernahme einer Führungsrolle. Unter Umständen könnte es hierbei sogar passieren, dass der*die Führende die Mittel $\mathrm{M}$ unbewusst einsetzt. Auch wenn die Absicht, Führung zu übernehmen, also nicht zwingend notwendig ist, so sollten Führende dennoch die Absicht haben, dass Geführte die Ziele Z und deren Umsetzung akzeptieren. Einfacher formuliert, sollten Führende beabsichtigen, Geführte zielorientiert zu beeinflussen.

\section{Zwischenmenschlicher Interaktionsprozess}

c) Die Mittel der Einflussnahme können sich dabei sowohl aus der Position einer Person ergeben (etwa Weisungsbefugnis, Gewährung von Beförderungen, Gehaltserhöhungen, Sonderzahlungen etc.) als auch aus den Mitteln der Persönlichkeit (Expertise, Charisma, Überzeugungskraft). Je nach Kontext bzw. Organisationstyp können dabei unterschiedliche Mittel eine unterschiedliche Relevanz haben, was auf die Verflechtungen der verschiedenen Dimensionen (hier Kontext und Interaktionsprozess) verweist. Hierdurch gelingt es $\mathrm{DF}_{\text {minimal }}$, Mittel der Führung sowohl in formal-hierarchischen Organisationen mit klar definierten Rollen als auch in Organisationen mit selbst organisierenden Teams und fluiden Rollenverteilungen zu erfassen (vgl. Laloux 2014).

d) Die Geführten müssen nicht allein wegen den Führenden die Ziele $\mathrm{Z}$ akzeptieren oder an deren Umsetzung mitwirken (vgl. Burns 1978). Andere Einflussfaktoren können ebenfalls eine Rolle spielen. Doch mindestens einer der beiden oder beide Fälle müssen hinzukommen, damit von einem Führungsprozess gesprochen werden kann: 1) Die Geführten hätten ohne die Führenden die Ziele $\mathrm{Z}$ bzw. deren Ausgestaltung bei sonst gleichen Bedingungen nicht akzeptiert. 2) Ohne den Führungsprozess würden die Geführten bei der Umsetzung der Ziele nicht in der gleichen Art und Weise mitwirken. Dies bedeutet auch, dass die Anwesenheit einer nominellen Führungskraft noch nicht zwangsläufig sicherstellt, dass Führung zustande kommt. So könnte eine Person, die eigentlich geführt werden soll, von sich aus bestimmte Ziele akzeptieren und umsetzen, beispielsweise weil sie besonders intrinsisch motiviert ist. Genauso ist es möglich, dass die Geführten zwar Führung brauchen, um die Ziele zu akzeptieren, dann aber eigenständig arbeiten oder dass die Ziele bereits von den Geführten akzeptiert werden, aber für die Umsetzung Führungsprozesse benötigt werden. Ein Beispiel dafür wäre politischer Aktivismus, wo die Teilnehmenden die Ziele häufig teilen, aber dennoch oftmals Führung not- 
wendig ist, um konstruktiv an der Umsetzung zu arbeiten.

e) Da nicht genauer bestimmt ist, welche Mittel M genutzt werden, um Geführte zu beeinflussen, können unterschiedliche Führungsstile mit $\mathrm{DF}_{\text {minimal }}$ in Einklang gebracht werden, sei es beispielsweise ein autoritärer, kooperativer oder partizipativer Führungsstil.

f) Die $\mathrm{DF}_{\text {minimal }}$ umfasst im Falle von Zielsetzung und Zielumsetzung alle Stufen von Partizipation der Geführten an der Gestaltung und Ausführung von Zielen (zu möglichen Stufen von Partizipation vgl. Stauffacher et al. 2008). So ist es möglich, dass die Geführten im Sinne einer Einwegkommunikation nur informiert oder befragt werden. Es können aber auch Austauschformate wie Kooperation oder Kollaboration gewählt werden, um eine Zielsetzung oder -umsetzung zu erreichen. Selbst die Übertragung der finalen Entscheidungsmacht auf die Geführten ist möglich, denn laut Minimaldefinition fällt Führung nicht zwingend mit formaler Entscheidungsmacht zusammen.

\section{Ziele Z}

g) $\mathrm{DF}_{\text {minimal }}$ fasst unter Führung sowohl das Setzen und Umsetzen von Zielen als auch die Beeinflussung von Personen. Somit umfasst sie die Führung von Institutionen (,Welche Ziele sind zu setzen?') als auch die Führung von Personen (, Wie gestaltet sich der interpersonelle Beziehungsprozess zwischen $\mathrm{X}$ und $\mathrm{Y}$ ?').

h) Die Ziele Z können weitreichend oder konkret, langfristig oder kurzfristig, vage oder klar definiert sein. So kann ein Ziel sowohl das umfassen, was in der Literatur oft als Sinn (,Purpose') oder Vision verstanden wird (vgl. Drucker 1973; Kirchgeorg et al. 2017) als auch enger gefasste Ziele, wie die Erreichung bestimmter Kennzahlen oder ein konkreter Arbeitsauftrag innerhalb einer Abteilung. Hiermit distanziert sich $\mathrm{DF}_{\text {minimal }}$ von jenen Führungsverständnissen, die nur dann von Führung sprechen wollen, wenn es um große, transformative, revolutionäre Ziele geht und Führung primär im politischen Bereich oder auf oberster Unternehmensführungsebene verorten (vgl. z. B. Rost 1991).

i) Im Hinblick auf die Setzung und Umsetzung von Zielen kann Führung nur im Bereich der Zielsetzung oder nur im Bereich der Zielumsetzung wirksam werden. So kann beispielsweise ein Ziel vorgegeben sein und die Aufgabe von Führung beschränkt sich darauf, dieses Ziel umzusetzen bzw. die Umsetzung zu gestalten. Von Gestaltung ist die Rede, wenn ein Ziel gegeben ist, die Umsetzung sich jedoch nicht unmittelbar aus der Zielformulierung ergibt. Dennoch sollte das Oder in $\mathrm{DF}_{\text {minimal }}$ nicht als exklusives Oder verstanden werden. Je nach Zuständigkeitsbereich können Führende sowohl bei der Ziel- setzung als auch bei der Zielumsetzung Führung übernehmen.

j) $\mathrm{DF}_{\text {minimal }}$ lässt auch unbestimmt, wessen Interessen die Ziele Z entsprechen. Die Ziele können den Interessen der Führenden, der Geführten oder aber denen einer Institution oder Dritter entsprechen oder aber die Interessen können miteinander harmonieren. So mag man es zwar als Ziel von Führung ansehen, dass die Interessen miteinander in Einklang gebracht werden, jedoch ist dies nach $\mathrm{DF}_{\text {minimal }}$ keine Voraussetzung dafür, um von Führung zu sprechen. ${ }^{7}$

k) Führende und Geführte können, müssen aber die Ziele $\mathrm{Z}$ nicht zwingend für sinnvoll halten, um $\mathrm{Z}$ zu akzeptieren. So können extrinsische Gründe, wie beispielsweise monetäre Anreize, für Z sprechen. Solche Gründe haben nicht damit zu tun, ob Führende oder Geführte $\mathrm{Z}$ als Ziel in einer Situation für das Richtige halten. Ein Ziel zu akzeptieren bedeutet demnach nicht zwangsläufig, von dem Ziel selbst überzeugt zu sein, sondern die Umsetzung des Ziels nicht zu verhindern und gegebenenfalls dessen Umsetzung zu unterstützen. Hierdurch gelingt es $\mathrm{DF}_{\text {minimal }}$ sowohl transaktionale als auch transformative Führung zu erfassen.

\section{Kontext K}

1) $\mathrm{DF}_{\text {minimal }}$ beschränkt Führung auf keinen bestimmten Lebensbereich. Somit kann sie Führung in den unterschiedlichsten Bereichen einfangen. Geführt wird u. a. in der Politik, in Bildungsinstitutionen, in gemeinnützigen Organisationen, in Vereinen und eben auch in Wirtschaftsunternehmen unterschiedlichster Größe.

m) Mit der Dimension des Kontextes öffnet $\mathrm{DF}_{\text {minimal }}$ auch den Blick für die Betrachtung der Rand- und Rahmenbedingungen von konkreten Führungsprozessen und deren Einfluss auf die in ihnen interagierenden Menschen. Rahmenbedingungen umfassen dabei einerseits die Gestaltung der Organisationen. So können sich Organisationen etwa in weichen Faktoren (Unternehmenskultur und Wertorientierungen) als auch in harten Faktoren (etwa Anreiz- und Leistungsmanagement) unterscheiden. ${ }^{8}$ Andererseits beziehen Rahmenbedingungen sich auch auf wirtschaftliche, gesellschaftliche und historische Bedingungen, in denen sich Organisationen verorten und umfassen dabei auch indirekte

\footnotetext{
${ }^{7}$ Für Burns (1978) ist z. B. die Harmonie der Interessen eine notwendige Bedingung für Führung.

${ }^{8}$ Die Spielmetapher hilft diese Unterscheidungen zu verstehen: Die konkreten Spielzüge der Führenden und Geführten sind immer eingebettet in das Spielverständnis und die Spielregeln einer Organisation und ihres äußeren Handlungskontextes (vgl. z. B. Suchanek 2015).
} 
oder nur imaginierte Einflussfaktoren, wie zum Beispiel die antizipierte Reaktion der Öffentlichkeit beim möglichen Bekanntwerden eines Skandals.

n) Der Kontext beeinflusst auch, welche Funktionen Führung erfüllen soll. Beispiele für Funktionen von Führung sind: Orientierung geben, Visionen erarbeiten, Einheit stiften, Aufgaben koordinieren, Prozesse moderieren, Mitarbeitende betreuen und fördern, Organisationsstrukturen gestalten oder die Organisation nach außen vertreten (vgl. dazu auch Maak/Pless 2006). Organisationstypen können sich darin unterscheiden, welche Funktionen sie von Führung und Führungspersonen erwarten und wie implizit bzw. explizit sie die Funktionen auf verschiedene Personen verteilen. (vgl. dazu auch Laloux 2014).

Die Adäquatheitsbedingung (2) fordert normative Neutralität. $\mathrm{DF}_{\text {minimal }}$ sollte also gute, gelungene ebenso wie (moralisch) schlechte Führung als Führung anerkennen. An dieser Stelle könnte man einwenden, dass $\mathrm{DF}_{\text {minimal }}$ dieser Adäquatheitsbedingung nicht gerecht wird, da sie Führung als Erfolgsbegriff auszeichnet: Man kann nur von Führung sprechen, wenn der Prozess der Einflussnahme zustande kommt, d. h., wenn $\mathrm{Y}$ durch $\mathrm{X}$ die Ziele $\mathrm{Z}$ akzeptiert und/ oder umsetzt. Hierdurch kann sie zumindest zwei Fälle schlechter Führung nicht erfassen. Der erste Fall ergibt sich, wenn X's Versuche der Zielvorgabe oder Anleitung der Umsetzung auf Seiten Y's nicht auf fruchtbaren Boden stoßen, der zweite, wenn man an $\mathrm{X}$ die Erwartung hat, $\mathrm{X}$ solle Führung übernehmen, jedoch keine Versuche unternimmt, $\mathrm{Y}$ zu beeinflussen. Zugegebenermaßen findet nach $\mathrm{DF}_{\text {minimal }}$ in beiden Fällen keine Führung statt. Im ersten Fall sollte man nach $\mathrm{DF}_{\text {minimal }}$ nicht von (schlechter) Führung, sondern höchstens von versuchter Führung sprechen. Im zweiten Fall wird X zwar eine Führungsaufgabe zugeschrieben, dieser kommt X jedoch nicht nach. Das Problem ist in diesem Falle nicht, dass $\mathrm{X}$ schlecht, sondern überhaupt nicht führt.

Immer wenn der Prozess der zielorientierten Einflussnahme zustande kommt, findet nach $\mathrm{DF}_{\text {minimal }}$ jedoch Führung statt. Dies eröffnet den Raum, um von gelungener ebenso wie von schlechter Führung als Führung zu sprechen. Daher erfüllt $\mathrm{DF}_{\text {minimal }}$ die Adäquatheitsbedingung (2).

Kommen wir nun zur Adäquatheitsbedingung (3). Diese Bedingung fordert, dass $\mathrm{DF}_{\text {minimal }}$ auf die ,Orte der Moral ${ }^{\text {* }}$ innerhalb von Führungsbeziehungen verweist. $\mathrm{DF}_{\text {minimal }}$ erfüllt diese Forderung, da jede der fünf Führungsdimensionen einen Ort für moralische Reflexionen und eine Möglichkeit, wo Führung aus moralischer Sicht gelingen oder auch scheitern kann, benennt:

(1) Im Hinblick auf die Führenden können sich u. a. folgende moralische Fragen stellen: Welche Rollenverantwortung haben die Führenden? Welche Rollen- erwartungen werden an sie gestellt? Welche Aufgaben bzw. Erwartungen sollten sie aus moralischer Sicht akzeptieren, welche zurückweisen? Welche moralisch wünschenswerten Charaktereigenschaften (Tugenden) bzw. nicht wünschenswerten Eigenschaften (Laster) haben die Führenden? Welche Wertüberzeugungen sollten sie besitzen? Und über welche moralischen Kompetenzen, wie beispielsweise Aufmerksamkeit, Urteilsfähigkeit und Umsetzungsfähigkeit, sollten sie verfügen?

(2) Da $\mathrm{DF}_{\text {minimal }}$ herausstellt, dass in Führungsprozessen Führende und Geführte miteinander interagieren, stellen sich entsprechende Fragen nach der (Rollen-)Verantwortung, den Tugenden, den Wertvorstellungen und den Kompetenzen auch in Bezug auf die Geführten.

(3) Der zwischenmenschliche Interaktionsprozess, der Führung auszeichnet, kann selbst moralisch betrachtet werden: Sind die Mittel M, die in einer Führungssituation gewählt werden, moralisch richtig bzw. akzeptabel? Welche Mittel wären moralisch richtig bzw. akzeptabel? Wie sollten bzw. dürfen Führende die Mittel aus moralischer Sicht einsetzen? Wie sollten bzw. dürfen Führende auf die Reaktion der Geführten bezüglich des Einsatzes der Mittel M reagieren? Da der Führungsprozess ein asymmetrischer Prozess ist, in dem die Geführten weniger (Wahl-)Freiheit besitzen und potentiell verletzlicher sind als die Führenden, stellt sich hier insbesondere die Frage, welche Mittel und welcher Mitteleinsatz die Freiheit der Geführten respektieren oder gar maximieren und ihre Verletzlichkeit beachten und nicht ausnutzen. Wie lässt sich der Interaktionsprozess gestalten, so dass Vertrauen zwischen Führenden und Geführten entsteht?

(4) Auch kann man die Ziele Z und deren Umsetzung moralisch bewerten, was zur vierten Dimension führt. Man kann beispielsweise fragen, ob die Ziele $\mathrm{Z}$ und deren Umsetzung moralisch richtig bzw. akzeptabel ist bzw. welche Ziele es ggf. wären. Verbunden hiermit stellt sich auch die Frage, ob es Stakeholder gibt, die durch die Ziele oder deren Umsetzung (unzulässigerweise) geschädigt werden. Auch kann man überlegen, welchen Werten die Ziele entsprechen, mit welchen Werten sie konfligieren oder welche sie ignorieren.

(5) Da der Führungsprozess nicht in einem ageschichtlichen, asozialen Raum stattfindet, kann außerdem auch der Kontext K moralisch evaluiert werden (vgl. z. B. Liden und Antonakis 2009). Welche kulturellen, rechtlichen oder institutionellen Rahmenbedingungen tragen dazu bei, dass eine Person Führung übernehmen kann bzw. über (wirksame) Mittel M verfügt? Werden hierdurch andere Personen benachteiligt? Welche Rahmenbedingungen begünstigen, dass Führende und Geführte sich im Führungsprozess moralisch richtig verhalten? 
Können die hinderlichen Rahmenbedingungen verändert werden? Wenn ja, wie und wer trägt hierfür Verantwortung? Wie sind die Rahmenbedingungen für neue Führungsprozesse zu gestalten, wo die feste, positionsgebundene Mittel der Einflussnahme wegfallen und personengebundene Mittel wichtiger werden?

Eine umfassende ethikorientierte Theorie der Führung sollte somit (zumindest) diese fünf Dimensionen und deren moralische Problemstellungen ansprechen. Hieraus ergibt sich auch eine klare Kritikmöglichkeit bestehender führungsethischer Theorien: Um als solche zu gelten, sollten sie alle fünf ,Orte der Moral ' berücksichtigen. Dabei muss eine Theorie nicht alle Dimensionen gleichermaßen moralisch beleuchten. Abhängig von ihrem Fokus und/oder ihrer Problemstellung kann es sinnvoll sein, dass sie sich auf ein oder zwei Dimensionen oder deren Wechselwirkungen fokussiert. Wichtig ist aber, dass diese Fokussierung einer bewussten und entsprechend ausgewiesenen Entscheidung folgt und nicht daraus resultiert, dass bestimmte Dimensionen der Führung bzw. , Orte der Moral ‘ übersehen wurden.

$\mathrm{DF}_{\text {minimal }}$ erfüllt auch die Adäquatheitsbedingung (4), da sie zwar die Dimensionen der Führung und die damit verbundenen ,Orte der Moral' erkennen lässt, sich jedoch auf keine Moraltheorie festlegt. $\mathrm{DF}_{\text {minimal }}$ kann als Grundlage für unterschiedliche moraltheoretischen Zugänge dienen. Abhängig von dem spezifischen moraltheoretischen Zugang mag man einzelne Dimensionen der Führung anders gewichten und unterschiedliche Fragen stellen. ${ }^{9}$ Dabei sollte jedoch der vorherige Punkt nicht vergessen werden: Eine führungsethische Theorie sollte keine Dimension und deren moralische Fragen übersehen oder unbegründet außenvorlassen.

Nachdem gezeigt wurde, dass $\mathrm{DF}_{\text {minimal }}$ die vier Adäquatheitsbedingungen erfüllt, ist abschließend wichtig zu betonen, dass $\mathrm{DF}_{\text {minimal }}$ sich nicht darauf festlegt, dass Führung

\footnotetext{
${ }_{9}$ Aus Sicht der Tugendethik mag sich die Frage nach den Tugenden von Führenden und Geführten aufdrängen und aus Sicht der Deontologie die nach den Pflichten und Rechten von Führenden und Geführten und wie man den Interaktionsprozess gestalten kann, so dass Geführte nicht bloß als Mittel zum Zweck gebraucht werden. Ein*e Fürsorgeethiker*in mag vor allem daran interessiert sein, wie im Führungsprozess Geführte als schwächere Parteien nicht geschädigt werden und sie in ihrer Entwicklung unterstützt werden können. Utilitarist*innen könnten sowohl im Hinblick auf den Interaktionsprozess als auch die Ziele und die Zielumsetzung das allgemeine Glück und die Interessen der betroffenen Stakeholder in dem Mittelpunkt stellen. Vertragstheoretiker*innen wiederum werden wahrscheinlich ihren Fokus auf den Kontext der Frage nach den geeigneten Rahmenbedingungen legen. Hier zeigt sich, dass gerade klassische Moraltheorien entsprechend in ihrer Auswahl einzelner Dimensionen zu motivieren bzw. zu erweitern sind, wenn sie als führungsethische Theorien hilfreich sein sollen.
}

nur oder primär moralisch bewertet werden sollte. Zwar kann man auf Basis von $\mathrm{DF}_{\text {minimal }}$ argumentieren, dass es geboten ist, Führung moralisch zu beleuchten, da Führung ein zwischenmenschlicher Prozess ist, der unterschiedliche menschliche Interessen tangiert, jedoch kann man Führung auch anhand primär nicht-moralischer Kriterien wie beispielsweise Effizienz oder Effektivität beurteilen. ${ }^{10}$ $\mathrm{DF}_{\text {minimal }}$ lässt offen, wie man die unterschiedlichen Kriterien gewichten und gegeneinander abwägen sollte. Dies ist wichtig, damit $\mathrm{DF}_{\text {minimal }}$ die Adäquatheitsbedingung (1) und (2) erfüllt. Damit kann DF ${ }_{\text {minimal }}$ das Versprechen einlösen, ein gemeinsames Verständnis als Konstante für verschiedene Diskussionen zur Führung zu bilden. Natürlich heißt das nicht, dass eine konkrete Führungsdiskussion hier nicht Stellung beziehen kann oder sollte.

\section{Analyse führungsethischer Theorien auf Basis der Minimaldefinition der ührung}

In diesem Abschnitt soll gezeigt werden, dass $\mathrm{DF}_{\text {minimal }}$ insbesondere dazu geeignet ist, auf Leerstellen im Hinblick auf die Dimensionen der Führung und die damit verbundenen moralischen Fragestellungen hinzuweisen, wenn man $\mathrm{DF}_{\text {minimal }}$ als allgemeinen Bezugsrahmen nimmt, vor dessen Hintergrund man führungsethische Theorien analysiert. Hierbei zeigt sich auch, dass $\mathrm{DF}_{\text {minimal }}$ Führung umfassender erfasst als andere Definitionen und Theorien. Um dies zu herauszuarbeiten, analysieren wir für die führungsethische Debatte einflussreiche Theorien vor dem Hintergrund von von $\mathrm{DF}_{\text {minimal }}$. Dass viele führungsethische Theorien nicht alle Dimensionen von Führung in ihren Definitionen gleichermaßen beachten, veranschaulicht überblicksartig Tabelle 1.

Da es im Zuge dieses Artikels nicht möglich ist, die Analysen der jeweiligen Theorien detailliert darzustellen, konzentrieren wir uns im Folgenden exemplarisch auf drei Theorien und zeigen auf, wie die Analyse auf Basis von $\mathrm{DF}_{\text {miminal }}$ aussehen kann. Die Rede ist von der Theorie der transformationalen Führung nach Bass, der Theorie der ethischen Führung und der verantwortungsvollen Führung.

\subsection{Transformationale Führung}

In seinem Buch Leadership definiert Burns Führung wie folgt: ,(...) leadership as leaders inducing followers to act for certain goals that represent the values and motivations

\footnotetext{
$\overline{10}$ Natürlich können Effizienz und Effektivität gerade beim Konsequentialismus auch moralisch relevant sein. Allerdings ist nicht jede Effizienz- bzw. Effektivitätsüberlegung zwingend konsequentialistisch oder überhaupt moralisch motiviert.
} 
Tabelle 1 Führungsethische Theorien und Dimensionen der Führung

\begin{tabular}{|c|c|c|c|c|c|}
\hline & 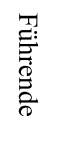 & $\underset{\substack{Q \\
\stackrel{0}{2}}}{\stackrel{Q}{2}}$ & 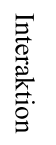 & $\frac{\stackrel{N}{\partial}}{\partial}$ & 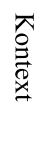 \\
\hline \multicolumn{6}{|l|}{ TRANSFORMATIONALE FÜHRUNG } \\
\hline $\begin{array}{l}\text { „Such leadership occurs when one or more person engage with others in such } \\
\text { a way that leaders and followers raise one another to higher levels of motivation } \\
\text { and morality." (Burns 1978: 21) }\end{array}$ & $\checkmark$ & $\checkmark$ & $\checkmark$ & $\checkmark$ & \\
\hline \multicolumn{6}{|l|}{ ETHISCHE FÜHRUNG } \\
\hline $\begin{array}{l}\text { "We define ethical leadership here as the demonstration of normatively } \\
\text { appropriate conduct through personal actions and interpersonal relationships, } \\
\text { and the promotion of such conduct to followers through a twoway } \\
\text { communication, reinforcement, and decision-making." (Brown et al. 2005: } \\
\text { 120) }\end{array}$ & $\checkmark$ & $(\sqrt{ })$ & $\checkmark$ & & $\checkmark$ \\
\hline \multicolumn{6}{|l|}{ VERANTWORTLICHE FÜHRUNG } \\
\hline $\begin{array}{l}\text { "In this context the purpose of leadership can be understood as to build and } \\
\text { cultivate sustainable and trustful relationships to different stakeholders inside } \\
\text { and outside the organization and to coordinate their action to achieve common } \\
\text { objectives (e. g. triple-bottom-line goals), business sustainability and } \\
\text { legitimacy and ultimately to realize to help a good (i.e. ethically sound) and } \\
\text { shared vision." (Maak und Pless 2006: 103) }\end{array}$ & $\checkmark$ & $\checkmark$ & $\checkmark$ & $\checkmark$ & $\checkmark$ \\
\hline \multicolumn{6}{|l|}{ AUTHENTISCHE FÜHRUNG } \\
\hline $\begin{array}{l}\text { "[We] define authentic leadership in organizations as a process that draws from } \\
\text { both positive psychological capacities and a highly developed organizational } \\
\text { context, which results in both greater self-awareness and self-regulated positive } \\
\text { behaviors on the part of leaders and associates, fostering positive self- } \\
\text { development." (Luthans und Avolio 2003: 243) }\end{array}$ & $\checkmark$ & $\checkmark$ & $\checkmark$ & & $\checkmark$ \\
\hline \multicolumn{6}{|l|}{ RELATIONALE FÜHRUNG } \\
\hline $\begin{array}{l}\text { „Leadership (is) a relational process co created by leaders and followers in } \\
\text { context.“ (Fairhurst und Uhl-Bien } 2012,1044 \text { ) }\end{array}$ & $\checkmark$ & $\checkmark$ & $\checkmark$ & & $\checkmark$ \\
\hline \multicolumn{6}{|l|}{ DIENENDE FÜHRUNG } \\
\hline $\begin{array}{l}\text { "The servant leader is servant first. (...) The difference manifests itself in the } \\
\text { care taken by the servant - first to make sure that other people's highest-priority } \\
\text { needs are being served (...). Do those served grow as persons? Do they, while } \\
\text { being served, become healthier, wiser, freer, more autonomous, more likely } \\
\text { themselves to become servants? And what is the effect on the least privileged } \\
\text { in society; will they benefit or, at least, not be further deprived?" (Greenleaf, } \\
\text { 1977: 13-14) }\end{array}$ & $\checkmark$ & $\checkmark$ & $\checkmark$ & $\checkmark$ & \\
\hline \multicolumn{6}{|l|}{ SPIRITUELLE FÜHRUNG } \\
\hline $\begin{array}{l}\text { "I define spiritual leadership as comprising the values, attitudes, and behaviors } \\
\text { are necessary to intrinsically motivate one's self and others so that they have a } \\
\text { sense of spiritual survival through calling and membership. This entails } \\
\text { 1. creating a vision wherein organization members experience a sense of calling } \\
\text { in that their life has meaning and makes a difference; } \\
\text { 2. establishing a social/organizational culture based on altruistic love whereby } \\
\text { leaders and followers have genuine care, concern, and appreciation for both } \\
\text { self and others, thereby producing a sense of membership and feel understood } \\
\text { and appreciated." (Fry 2003: 694-695) }\end{array}$ & $\checkmark$ & $\checkmark$ & $\checkmark$ & $\checkmark$ & $\checkmark$ \\
\hline
\end{tabular}

- the wants and needs, the aspirations and expectationsof both leaders and followers" (Burns 1978). Im Hinblick auf die Frage, wie Führende Geführte zielorientiert beeinflussen, unterscheidet Burns zwischen transaktionaler und transformationaler Führung. Bei transaktionaler Führung werden Geführte dazu motiviert, ein Ziel zu akzeptieren, weil sie hierdurch etwas anderes für sich als wertvoll Erachtetes bekommen. Bei transformationaler Führung geht es im Gegensatz dazu darum, dass Führende und Geführte gemeinsame Ziele verfolgen, von denen sie beide überzeugt sind: „Such leadership occurs when one or more person engage with others in such a way that leaders and followers raise one another to higher levels of motivation and morality" (ebd.). Transformationale Führung zielt somit auf moralische Entwicklung und Verbesserung ab (vgl. ebd.).

Bass und seine Kollegen greifen auf Burns Theorie der transformationalen Führung zurück, modifizieren und erweitern diese jedoch. So abstrahieren sie zum einen von 
einem zentralen Aspekt von Burns Theorie: Transformationale Führung muss nicht mehr (ausschließlich) das Ziel verfolgen, Geführte auf eine höhere moralische Entwicklungsstufe zu bringen. Vielmehr kann transformationale Führung eingesetzt werden, um unterschiedliche Ziele zu verwirklichen (vgl. Bass und Riggo 2006).

Auch buchstabieren Bass und Kollegen aus, wie transformationale Führung gelingen kann. Sie identifizieren vier Komponenten transformationaler Führung (vgl. ebd.): Erstens verfügen Führende, durch ihr Vorbild, aber auch durch ihnen zugeschriebene Eigenschaften, über Einfluss auf die Geführten (,idealized influence'). Zweitens sind die Führenden in der Lage sowohl konkrete Ziele als auch Visionen inspirierend und motivierend zu vermitteln (,inspirational motivation'). Drittens fordern Führende die Geführten intellektuell heraus und ermutigen sie neue und kreative Lösungen für Probleme zu finden (,intellectual stimulation'). Viertens betrachten Führende Geführte als Individuen und wollen sie individuell fördern (,individualized consideration").

Bass und Riggo konzentrieren sich jedoch nicht nur auf transformationale Führung, sondern entwickeln ein ,FullRange-Leadership-Modell' (vgl. ebd.). So beschreiben sie, dass Führende Geführte nicht nur transformational beeinflussen, sondern teilweise auf kontingente Belohnungen und teilweise auf aktives oder passives Management-by-Expectation zurückgreifen. Verzichten Führende darauf zu führen, treffen sie keine Entscheidungen und halten sich bewusst heraus, sprechen Bass und Riggo von Laissez-Faire Leadership. Auch wenn Bass und Riggo das ,Full-Range-Leadership-Modell' formulieren, so wollen sie keine grundlegende Definition von Führung erarbeiten. Dies sieht man allein schon daran, dass sie ihre Ausführungen normativ nicht neutral sind. Sie sprechen sich eindeutig für einen transformationalen Führungsstil aus. Dieser sei die effektivste Art zu führen (vgl. ebd.).

Eine strittige Frage ist, ob und inwieweit transformationale Führung zugleich moralisch gute Führung ist. Bass und Steidlmeier verweisen in ihrer Diskussion dieser Frage auf drei ,Orte der Moral': „The ethics of leadership rests upon three pillars: (1) the moral character of the leader; (2) the ethical legitimacy of the values embedded in the leaders vision, articulation, and program which followers either embrace or reject; and (3) the morality of the processes of social ethical choice and action that leaders and followers engage in and collectively pursue" (Bass und Steidlmeier 1999). Bass und Kollegen unterscheiden nun zwischen pseudo-transformationaler und authentischer transformationaler Führung. Authentische transformationale Führung wird nur erreicht, wenn einerseits der Charakter der Führenden vorbildhaft ist und die vier Komponenten transformationaler Führung ernsthaft und (auch) zum Nutzen der Geführten umgesetzt werden. (vgl. ebd.). Gerade im
Hinblick auf die Komponente der individuellen Betrachtung argumentieren Bass und Kollegen aber, dass diese letztendlich nur bei authentischer Führung verwirklicht sei, weshalb sie transformationale Führung und authentische transformationale Führung letztendlich mehr oder weniger gleichsetzen (vgl. Bass und Steidlmeier 1999; Bass und Riggo 2006). Sie arbeiten jedoch auch explizit heraus, dass auch transaktionale Führung aus moralischer Sicht gute Führung sein kann (vgl. Bass und Steidlmeier 1999).

Betrachtet man Bass Verständnis von transformationaler Führung vor dem Hintergrund von $\mathrm{DF}_{\text {minimal }}$, ist zunächst zu bemerken, dass transformationale Führung unter den Führungsbegriff von $\mathrm{DF}_{\text {minimal }}$ fällt. Transformationale Führung wird auch als zielorientierter und asymmetrischer Prozess der Einflussnahme zwischen Führenden und Geführten verstanden. Bass und Kollegen sprechen in ihren Ausführungen nun alle fünf Dimensionen der Führung an, jedoch thematisieren sie nur die Dimension der Führenden, die der Ziele und der Zielumsetzung und des Interaktionsprozesses als ,Orte der Moral', ohne dass sie dies ausreichend begründen. Dies muss man auf Basis von $\mathrm{DF}_{\text {minimal }}$ als Manko ansehen, auch wenn eine Offenheit gegenüber einer erweiterten Betrachtung, die die fehlenden Dimensionen einschließt, besteht.

\subsection{Ethische Führung}

Wenden wir uns nun zwei Theorien zu, die dezidiert eine Theorie moralisch guter Führung formulieren wollen. Den Anfang macht die Theorie der ethischen Führung, wie sie von Brown, Treviño und Harrison vorgebracht wird. Sie stellen folgende Definition zur Diskussion: „We define ethical leadership here as the demonstration of normatively appropriate conduct through personal actions and interpersonal relationships, and the promotion of such conduct to followers through a two-way communication, reinforcement, and decision-making" (Brown et al. 2005). Mit ihrer Definition heben sie drei Aspekte hervor: (1) Ethische Führung basiert auf sozialen Lernprozessen, in denen Führende zu moralischen Vorbildern für Geführten werden und diese zur Nachahmung anregen. (2) Anreiz- und Bestrafungssysteme müssen so ausgestaltet werden, dass sie ethisches Verhalten fördern. (3) Die Wichtigkeit von ethischen Themen muss explizit und nachdrücklich kommuniziert werden.

Da die Autor*innen explizit keine grundlegende Führungsdefinition formulieren wollen, sondern eine Definition ethischer Führung, müssen wir nicht hinterfragen, ob ihre Definition weit genug gefasst ist, um unterschiedliche Führungsverständnisse einzufangen, oder normativ neutral ist. Da Brown, Treviño und Harrison Führung als einen interpersonalen und asymmetrischen Prozess der Einflussnahme verstehen, der zwischen Führenden und Geführten 
stattfindet, fällt ethische Führung unter den von $\mathrm{DF}_{\text {minimal }}$ vorgebrachten Führungsbegriff.

Im Hinblick auf $\mathrm{DF}_{\text {minimal }}$ stellt sich jedoch die Frage, ob die Autor*innen alle ,Orte der Moral ' bedacht haben. Unstrittig erscheint, dass sie die Dimension der Führenden, des interpersonellen Interaktionsprozesses und des Kontexts beachten. Die Dimension der Führenden rückt durch deren Vorbildfunktion in den Mittelpunkt. Auch untersuchen die Autoren, welche individuellen Eigenschaften sich positiv auf ethisches Führungsverhalten auswirken und welche Tugenden ethischen Führenden von Geführten attestiert werden (vgl. Brown et al. 2005; Brown und Treviño 2006). Durch den Fokus auf die sozialen Lernprozesse, die Vorbildfunktion der Führenden und die positive bzw. negative Sanktionierung von Verhalten von Geführten ist auch die Dimension des interpersonellen Interaktionsprozesses mitgedacht. Des Weiteren thematisieren die Autor*innen an unterschiedlichen Stellen auch den Kontext, in dem Führung stattfindet. So sprechen sie beispielsweise von normativ angemessenem Verhalten und verweisen darauf, dass es sich aus der jeweiligen historischen und sozio-kulturellen Situation ergibt, welches Verhalten als normativ angemessen gilt (vgl. Brown et al. 2005). Außerdem weisen Brown und Treviño darauf hin, dass ethische Führung positiv damit korreliert ist, ob man ethische Rollenmodelle in seiner Karriere identifizieren konnte und ob das Unternehmensklima und die Unternehmenskultur ethisches Verhalten positiv unterstützt (vgl. Brown und Treviño 2006).

Die Dimension der Geführten wird jedoch nicht im gleichen Maße mitbedacht. Zwar untersuchen die Autor*innen, welche Auswirkung ethische Führung auf das moralische Verhalten und deren Motivation und Zufriedenheit hat, jedoch wird nicht gefragt, welche moralischen Anforderungen an die Geführten gestellt werden können. Man kann die Theorie der ethischen Führung dahingehend kritisieren, dass sie die Dimension der Führenden deutlich mehr beleuchtet als die der Geführten. Doch es besteht noch eine zweite Schwierigkeit: Durch die Fokussierung auf soziales Lernen und die Vorbildfunktion der Führungskraft gerät der Aspekt der Zielsetzung und -umsetzung in den Hintergrund. Es wird zwar als Ziel für Führende angesehen, moralisches Verhalten bei den Geführten zu fordern, jedoch wird nicht thematisiert, welche Ziele verfolgt werden und wie diese umgesetzt werden. Die Frage, welche Ziele moralisch akzeptabel und richtig sind, wird nicht angesprochen, was man als große Leerstelle der Theorie der ethischen Führung ansehen kann.

\subsection{Verantwortungsvolle Führung}

Auch Maak und Pless wollen in ihrem Artikel „Responsible Leadership in a Stakeholder Society" keine grundlegende Führungsdefinition, sondern eine führungsethische Theorie formulieren. Ansatzpunkt ihrer Überlegungen ist eine von ihnen beobachtete Engführung von Führung auf das Verhältnis Führungskraft-Mitarbeiter*in. Sie wollen den Fokus erweitern (vgl. Maak und Pless 2006; Pless und Maak 2011). Führung in einer modernen und vernetzten Welt bedeutet, mit verschiedensten Stakeholdern (Mitarbeiter*innen, Aktionär*innen, Kund*innen, Geschäftspartner*innen, aber auch der Gesellschaft oder Umwelt) zu interagieren. Das Ziel verantwortungsvoller Führung beschreiben die Autoren wie folgt: „In this context the purpose of leadership can be understood as to build and cultivate sustainable and trustful relationships to different stakeholders inside and outside the organization and to co-ordinate their action to achieve common objectives (e.g., triple-bottom-line goals), business sustainability and legitimacy and ultimately to realize to help a good (i.e. ethically sound) and shared vision" (Maak und Pless 2006).

Da Maak und Pless Führung als sozial-relationales, ethisches Phänomen beschreiben, das zwischen Führenden und einer breiten Gruppe von Geführten stattfindet, und darauf ausgerichtet ist, dass die Gruppen eine gemeinsame und moralisch solide Vision akzeptieren und umsetzen (vgl. Maak/Pless 2006; Pless und Maak 2011), fällt verantwortungsvolle Führung unter den Führungsbegriff von $\mathrm{DF}_{\text {minimal }}$. Führende und unterschiedliche Stakeholder nehmen dabei nicht zwangsläufig unterschiedliche hierarchische Positionen ein, sondern interagieren teilweise auf gleicher Augenhöhe (vgl. Maak und Pless 2006). Damit verliert jedoch die Führungsbeziehungen, von denen Maak und Pless sprechen, nicht an ihrer Asymmetrie. So betonen die Autoren, dass die Aufgabe von Führenden folgende sei: „They are weavers who bring together different people to follow a shared and morally sound vision. They are facilitators of relational processes of co-creation and orchestrators for achieving common objectives" (ebd.). Um dieser Aufgabe gerecht werden zu können, müssen Führende unterschiedliche Rollen einnehmen können: Steward, Bürger*in, Visionär*in, Diener*in, Coach, Architekt*in, Geschichtenerzähler*in, Erneuer*in (vgl. ebd.).

Wie diese Ausführungen zeigen, bedenken Maak und Pless die Dimension der Führenden und fragen gezielt danach, welche Rollen sie beherrschen und welche (moralischen) Eigenschaften sie besitzen sollten. So fordern sie insbesondere, dass Führende relationale Intelligenz, also emotionale und ethische Intelligenz besitzen (vgl. ebd.). Auch die Dimension der Geführten wird angesprochen und die aktive Rolle der Geführten wird betont, jedoch bleibt diese aus moralischer Sicht unterbeleuchtet. Es wird nicht gefragt, welche Rollen Geführte beherrschen oder welche Eigenschaften sie besitzen sollten. Die Dimension der Interaktion wird explizit als moralischer Ort ausgewiesen, da Führung als moralischer, werte-basierter und normativer Prozess beschrieben und die Frage aufgeworfen wird, wie 
Ziele erreicht werden (vgl. ebd.). Die Autoren fordern im speziellen, dass die Interaktionen so gestaltet werden sollten, dass sie vertrauensvoll, nachhaltig und fair sind und alle Akteur*innen als gleiche und verletzliche Wesen respektiert werden (vgl. Maak und Pless 2006; Pless und Maak 2011). Indem es als Aufgabe von verantwortlicher Führung angesehen wird, gemeinsame, nachhaltige und legitime Unternehmensziele zu verwirklichen und eine moralisch gute Unternehmensvision zu entwickeln, wird deutlich, dass auch die Dimension der Ziele und Zielumsetzung beachtet wird. Gleiches gilt für die letzte Dimension der Führung, den Kontext. So beginnen die Autoren ihren Artikel gleich mit einem Verweis auf die VUCA-Welt und die Schwierigkeiten, die sich hieraus für Führung ergeben (vgl. Maak und Pless 2006). So überrascht es nicht, dass sich die vier Grundherausforderungen für Führung aus dem jeweiligen Kontext ergeben. Es gilt somit kontextspezifisch zu überlegen, wer die Stakeholder sind, welche Wertvorstellungen sie haben und wie unternehmerisches Handeln in solch einem Kontext gelingen kann (vgl. ebd.). Die Theorie der verantwortlichen Führung überzeugt somit, da sie alle fünf relevanten, Orte der Moral' anspricht.

Es fallen jedoch zwei Engführungen auf. Einmal beschränken die Autoren sich auf Führung im Wirtschaftsbereich (vgl. ebd.). Hiermit lassen sie offen, wie sie (verantwortungsvolle) Führung in anderen Kontexten beschreiben würden (vgl. ebd.). Zweitens wird Führung teils explizit, teils implizit ausschließlich ,groß ' gedacht. Die Beispiele drehen sich um internationale Manager*innen und Firmenlenker*innen. Damit bleibt zumindest unterbelichtet, dass Führung auch im Kleinen, in Arbeitsgruppen, Laboren, Tutorien geschieht, wo viele der von ihnen angebrachten Rollenbilder, sei es beispielsweise der*die Visionär*in, oder Architekt*in, nicht zum Tragen kommen. Maak und Pless verbinden in ihrem Ansatz damit zu strikt formale und herausgehobene Führungspositionen mit Führungsverantwortung und Führungshandeln. Hier zeigt sich die Stärke von $\mathrm{DF}_{\text {minimal }}$, die Führung, Führende und Geführte dort verortet, wo die Kriterien zutreffen, unabhängig von Position oder Zielvorstellung. Damit lassen sich auch Führungskräfte im mittleren Management und informelle Führungsprozesse beschreiben.

\section{Konklusion}

Dieser Artikel hat es sich zum Ziel gemacht, eine Minimaldefinition von Führung zu erarbeiten, um bestehende Antworten auf die Frage nach guter Führung zu beurteilen, miteinander zu vergleichen und die Basis für eine zusammenführende Führungstheorie zu legen. Damit die Minimaldefinition ihre diskussionsstrukturierende Aufgabe erfüllen kann, muss sie unterschiedliche Führungsverständnisse und
Führungstheorien erfassen, selbst normativ neutral sein, aufzeigen, welche Dimensionen von Führung moralisch relevant sind und sich dabei noch auf keine Moraltheorie festlegen.

Im Abschnitt 2 wurde gezeigt, dass $\mathrm{DF}_{\text {minimal }}$ diese Kriterien erfüllt. $\mathrm{DF}_{\text {minimal }}$ definiert Führung als zielorientierten, asymmetrischen und kontextsensiblen Prozess der Einflussnahme zwischen Führenden und Geführten. Laut $\mathrm{DF}_{\text {minimal }}$ hat Führung eine interaktionsbezogene Komponente ((1) Führende, (2) Geführte und (3) zwischenmenschlicher Interaktionsprozess), eine zielbezogene Komponente ((4) Zielfindung, und -umsetzung) und eine systembezogene Komponente ((5) Kontext). Jede dieser fünf Dimensionen verweist auf einen Ort der moralischen Reflexion im Bereich der Führung.

Die Orte der moralischen Reflexion erschöpfen sich jedoch nicht in den fünf voneinander unterschiedenen Dimensionen. Die meisten Führungsprozesse in der Praxis umfassen mehr als eine der fünf Dimensionen. Dementsprechend gibt es Intersektionen, die wieder eigene, Orte der Moral ' hervorbringen. Ein Beispiel ist der Fall eines Interaktionsprozesses zwischen Führenden und Geführten. In der Interaktion realisiert sich die prinzipiell vorhandene Machtasymmetrie zwischen Führenden und Geführten zu einer ungleichen Verteilung von Freiheit und Vulnerabilität. Dies ist nur im Zusammenspiel der drei Dimensionen voll zu erfassen. Denn ob und inwiefern sich eine grundsätzlich vorhandene Asymmetrie zwischen Führendem und Geführten in der Praxis realisiert, liegt an der Ausgestaltung des Interaktionsprozesses (vgl. dazu Vondermaßen, Fröhlich, Schmalzried 2021).

Im Abschnitt 3 haben wir gezeigt, dass $\mathrm{DF}_{\text {minimal }}$ so konzipiert ist, dass mit ihr die fehlenden oder zu wenig beachteten moralischen Dimensionen in untersuchten Führungsdefinitionen klarer herausgearbeitet werden können. Dies wurde exemplarisch an prominenten führungsethischen Theorien aufgezeigt. Dabei zeigte sich, dass Bass und Kollegen in ihrer Theorie der transformationalen Führung nur die Dimension der Führenden, des Interaktionsprozesses und der Ziele als ,Orte der Moral' im Führungsbereich auszeichnen und die Theorie der ethischen Führung die Dimension der Geführten und der Ziele zu wenig beachten. Im Gegensatz dazu wurde mit Hilfe von $\mathrm{DF}_{\text {minimal }}$ deutlich, dass die Theorie der verantwortungsvollen Führung alle fünf Dimensionen der moralischen Reflexion mitbedenkt und somit in diesem Vergleich am überzeugendsten erscheint.

Die Erkenntnisse der vorliegenden Kurzanalysen sind selbstverständlich nicht hinreichend für eine abschließende Bewertung der untersuchten Theorien. Sie zeigen aber auf, wie eine Beurteilung möglich ist: Wenn $\mathrm{DF}_{\text {minimal }}$ die notwendigen Elemente von Führung akkurat beschreibt, dann ist die unmotivierte Nichtbeachtung einer Dimension ein Versäumnis. $\mathrm{DF}_{\text {minimal }}$ liefert dementsprechend einen 
grundlegenden Beitrag zur begrifflichen Schärfung der Führungsdebatte, indem die Definition theorieunabhängig und normativ neutral alle relevanten Dimensionen von Führung aufzeigt.

Damit zeigt $\mathrm{DF}_{\text {minimal }}$, dass Führung ein komplexer Prozess ist und als solcher nicht unterkomplex behandelt werden soll. Durch $\mathrm{DF}_{\text {minimal }}$ können wir der Komplexität in einfacher Weise gerecht werden: Wir können die verschiedenen Dimensionen von Führung analytisch getrennt identifizieren und sie als Orte der Moral erkennen. Gleichzeitig lassen sich so auch die Verflechtungen der verschiedenen Dimensionen klar erkennen und alle Dimensionen im Gesamtbild von Führung verorten. Damit gelingt es $\mathrm{DF}_{\text {minimal }}$ eine diskussionsstrukturiende Funktion zu erfüllen und so die Anschlussfähigkeit verschiedener Theorien zu ermöglichen. Zugleich ist durch $\mathrm{DF}_{\text {minimal }}$ die Basis gelegt, um in einem nächsten Schritt tiefer in die normative Debatte über gute Führung einzusteigen.

Funding Research Project Leadership Ethics funded by the Carl Zeiss Foundation.

Data availability Not applicable.

Code availability Not applicable.

\section{Declarations}

Conflict of interest Not applicable.

Open Access Dieser Artikel wird unter der Creative Commons Namensnennung 4.0 International Lizenz veröffentlicht, welche die Nutzung, Vervielfältigung, Bearbeitung, Verbreitung und Wiedergabe in jeglichem Medium und Format erlaubt, sofern Sie den/die ursprünglichen Autor(en) und die Quelle ordnungsgemäß nennen, einen Link zur Creative Commons Lizenz beifügen und angeben, ob Änderungen vorgenommen wurden. Die in diesem Artikel enthaltenen Bilder und sonstiges Drittmaterial unterliegen ebenfalls der genannten Creative Commons Lizenz, sofern sich aus der Abbildungslegende nichts anderes ergibt. Sofern das betreffende Material nicht unter der genannten Creative Commons Lizenz steht und die betreffende Handlung nicht nach gesetzlichen Vorschriften erlaubt ist, ist für die oben aufgeführten Weiterverwendungen des Materials die Einwilligung des jeweiligen Rechteinhabers einzuholen. Weitere Details zur Lizenz entnehmen Sie bitte der Lizenzinformation auf http://creativecommons.org/licenses/ by/4.0/deed.de.

\section{Literaturverzeichnis}

Avolio BJ, Gardner WL (2005) Authentic leadership development: getting to the root of positive forms of leadership. Leadersh Q 16:315-338. https://doi.org/10.1016/j.leaqua.2005.03.001

Bass B (1985) Leadership and performance beyond expectations. Free Press

Bass B, Riggio R (2006) Transformational leadership. Lawrence Erlbaum Associates
Bass B, Steidlmeier P (1999) Ethics, character, and authentic transformational leadership behavior. Leadersh Q 10(2):181-217. https://doi.org/10.1016/S1048-9843(99)00016-8

Beck K et al (2001) Manifesto for agile software development. https://agilemanifesto.org/iso/de/manifesto.html

Breidenbach J, Rollow B (2019) New work needs inner work. Verlag Franz Vahlen

Brown ME, Treviño LK (2006) Ethical leadership: a review and future directions. Leadersh Q 17(6):595-616. https://doi.org/ 10.1016/j.leaqua.2006.10.004

Brown ME, Treviño LK, Harrison DA (2005) Ethical leadership: a social learning perspective for construct development and testing. Organ Behav Hum Decis Proc 97(2):117-134. https://doi. org/10.1016/j.obhdp.2005.03.002

Burns J (1978) Leadership. Harper \& Row

Carnap R (1958) Induktive Logik und Wahrscheinlichkeit. Springer Verlag. https://doi.org/10.1007/978-3-7091-3142-8

Ciulla J (1995) Leadership ethics: mapping the territory. Bus Ethics Q 5:5-28. https://doi.org/10.2307/3857269

Drucker P (1973) Management tasks. Responsibilities Practices. Harper \& Row

Fairhurst GT, Uhl-Bien M (2012) Organizational discourse analysis (ODA) examining leadership as a relational process. Leadersh Q 23(6):1043-1062. https://doi.org/10.1016/j.leaqua.2012.10.005

Faix WG, Windisch L, Kisgen S et al (2020) A new model for stateof-the-art leadership education with performance as a driving factor for future viability. Leadersh Educ Personal Interdiscip J 2:59-74. https://doi.org/10.1365/s42681-020-00011-4

Forst R (2015) Normativität und macht. Suhrkamp

French J, Raven B (1968) The bases of social power. In: Cartwright D, Zander AF (eds) Group dynamics: research and theory. Harper \& Row, pp 259-269

Frey D, Schmalzried L (2013) Philosophie der Führung. Gute Führung lernen von Kant, Aristoteles. Popper \& Co, Springer

Fry LW (2003) Toward a theory of spiritual leadership. Leadersh Q 14(6):693-727

Greenleaf R (1977) Servant leadership. a journey into the nature of legitimate power and greatness. Paulist Press

Greenleaf R (2013) Selections from servant leadership. a journey into the nature of legitimate power and greatness. In: Ciulla JB, Uhl-Bien M, Werhane PHS (eds) Leadership ethics, vol 1. Sage, London, pp 103-108

Kort ED (2008) What, after all, is leadership? 'Leadership' and plural action. Leadersh Q 19(4):409-425. https://doi.org/10.1016/j. leaqua.2008.05.003

Laloux F (2014): Reinventing Organizations. Nelson Parker, Brüssel

Lemoine GJ, Hartnell CA, Leroy H (2019) Taking stock of moral approaches to leadership: an integrative review of ethical, authentic, and servant leadership. Acad Manag Ann 13(1):148187. https://doi.org/10.5465/annals.2016.0121

Liden RC, Antonakis J (2009) Considering context in psychological leadership research. Hum Relat 62(11):1587-1605. https://doi. org/10.1177/0018726709346374

Luthans F, Avolio BJ (2003) Authentic leadership. a positive developmental approach. In: Cameron KS, Dutton JE, Quinn RE (eds) Positive organizational scholarship. Barrett-Koehler, pp 241-263

Maak T, Pless N (2006) Responsible leadership in a stakeholder society - a relational perspective. J Business Ethics 66(1):99115. https://doi.org/10.1007/s10551-006-9047-z

Mackey JD, Ellen BP III, McAllister CP, Alexander KC (2020) The dark side of leadership: a systematic literature review and metaanalysis of destructive leadership research. J Bus Res. https:// doi.org/10.1016/j.jbusres.2020.10.037 
Pless N, Maak T (2011) Responsible leadership pathways to the future. J Bus Ethics 98(1):3-13. https://doi.org/10.1007/ s10551-011-1114-4

Rost J (1991) Leadership in the twenty-first century. Praeger

Rost J (1995) Leadership a discussion about ethics. Bus Ethics Q 5(1):129-142. https://doi.org/10.2307/3857276

Rost J (1998) Leadership concepts and theories in organizations. In: Hickmann GR (ed) Leading organizations. Perspectives for a new era. Sage Publications Ltd, pp 98-114

Schyns B, Schilling J (2013) How bad are the effects of bad leaders? A meta-analysis of destructive leadership and its outcomes. Leadersh Q 24(1):138-158. https://doi.org/10.1016/j.leaqua. 2012.09.001

Stauffacher M, Flüele T, Krütli P, Scholz RW (2008) Analytic and dynamic approach to collaboration. A transdisciplinary case study on sustainable landscape development in a swiss prealpine region. Syst Pract Action Res 21:409-422. https://doi.org/10. 1007/s11213-008-9107-7

Suchanek A (2015) Unternehmensethik. In Vertrauen investieren, Mohr Siebeck, Tübingen

Van Dierendonck D (2011) Servant leadership a review and synthesis. J Manag 37(4):1228-1261

Vondermaßen M, Fröhlich F, Schmalzried L (2021) Leadership dimensions. https://youtu.be/n4AGXhE8HtU (letzter Besuch: 29.09.2021)

Winston BE, Patterson K (2006) An integrative definition of leadership. Int J Leadersh 1(2):6-66 